\title{
Synergistic effect of kaempferol and 5-fluorouracil on the growth of colorectal cancer cells by regulating the PI3K/Akt signaling pathway
}

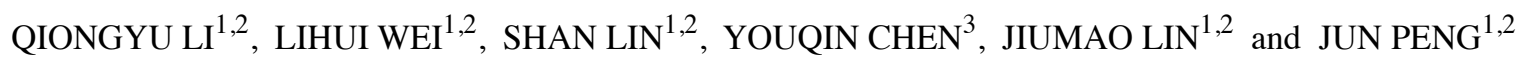 \\ ${ }^{1}$ Academy of Integrative Medicine; ${ }^{2}$ Fujian Key Laboratory of Integrative Medicine on Geriatrics, \\ Fujian University of Traditional Chinese Medicine, Fuzhou, Fujian 350122, P.R. China; ${ }^{3}$ Case Western Reserve \\ University School of Medicine, Rainbow Babies and Children's Hospital, Cleveland, OH 44106, USA
}

Received November 21, 2018; Accepted April 24, 2019

DOI: $10.3892 / \mathrm{mmr} .2019 .10296$

\begin{abstract}
Combination chemotherapy with chemosensitizers can exert synergistic therapeutic effects, reduce toxicity, and delay the induction of drug resistance. In the present study, the antitumor effects were investigated, and the possible underlying mechanisms of kaempferol combined with 5-fluorouracil (5-FU) in colorectal cancer cells were explored. HCT-8 or HCT-116 cells were treated with various concentrations of kaempferol and/or 5-FU for the indicated time-points. An MTT assay was used to determine cell viability, whereas the synergistic effects were assessed by calculating the combination indices of kaempferol and 5-FU. Annexin V analysis and Hoechst staining were used to determine cell apoptosis. q-PCR and western blotting were performed to determine the expression levels of Bax, Bcl-2, thymidylate synthase (TS), PTEN, $\mathrm{PI} 3 \mathrm{~K}, \mathrm{AKT}$, and p-AKT. The combination of kaempferol and 5-FU was determined to be more effective in inhibiting cell viability than either of the agents alone. The inhibition of tumors in response to kaempferol and 5-FU was associated with the reduction in proliferation ability and stimulation of apoptosis. The protein results indicated that kaempferol and
\end{abstract}

Correspondence to: Dr Jiumao Lin or Dr Jun Peng, Academy of Integrative Medicine, Fujian University of Traditional Chinese Medicine, 1 Qiuyang Road, Minhou Shangjie, Fuzhou, Fujian 350122, P.R. China

E-mail: linjiumao@fjtcm.edu.cn

E-mail: pjunlab@hotmail.com

Abbreviations: CRC, colorectal cancer; 5-FU, 5-fluorouracil; TS, thymidylate synthase; MTT, 3-(4,5-dimethyl-thiazol-2-yl)-2, 5-diphenyltetrazolium bromide; FBS, fetal bovine serum; FACS, fluorescence-activated cell sorting; SDS-PAGE, sodium dodecyl sulfate-polyacrylamide minigel; DMSO, dimethyl sulfoxide; PI3K, phosphatidylinositol-3-kinase; AKT, protein kinase B; CI, combination index

Key words: colorectal cancer, synergistic effect, kaempferol, 5-fluorouracil, drug resistance, PI3K/Akt
5-FU could significantly upregulate the expression levels of Bax and downregulate the expression levels of Bcl-2 and TS. Furthermore, the combination treatment greatly inhibited the activation of the PI3K/Akt pathway, suggesting the involvement of this pathway in the synergistic effects. The present study demonstrated that kaempferol has a synergistic effect with 5-FU by inhibiting cell proliferation and inducing apoptosis in colorectal cancer cells via suppression of TS or attenuation of p-Akt activation. The combination of kaempferol and 5-FU may be used as an effective therapeutic strategy for colorectal cancer.

\section{Introduction}

Colorectal cancer (CRC) is a major public health problem since it is the third most commonly diagnosed cancer resulting in mortality worldwide (1). Moreover, the incidence rates of colorectal cancer in developing countries, including China, have risen due to the growth of the aging population and adoption of westernized behaviors and lifestyles. Surgery and adjuvant chemotherapy are the main treatments for colorectal cancer. However, 40-50\% of patients succumb to this disease due to recurrence, metastases, and drug resistance $(2,3)$. In addition, severe side effects caused by chemotherapeutic agents lead to the deterioration of the quality of life of patients. Therefore, it is necessary to develop tolerable treatment strategies with increased sensitivity in order to improve the clinical outcome and overall survival rates.

The most widely used chemotherapeutic drug, 5-fluorouracil (5-FU), is a first-line base treatment of colorectal cancer (4-6). 5-FU inhibits cancer cell growth and initiates apoptosis by inducing DNA damage during replication and hindering its repair. It could disturb the synthesis of the pyrimidine thymidine, a nucleoside required for DNA replication, and block the activity of thymidylate synthase (TS) (7). Although 5-FU treatment has been demonstrated to be effective for CRC, it is associated with severe side effects and acquired drug resistance $(8,9)$. Therefore, further studies are required to identify agents that can increase the efficacy of 5-FU and reduce its side effects. 
Clinically, patients with low TS expression in tumor tissues exhibit improved response to 5-FU-based therapy, indicating that TS expression may be involved in the development of 5-FU resistance. A previous study revealed that cancer cells acquire 5-FU resistance when stimulated with low doses of 5-FU for a prolonged period, which is accompanied by high expression levels of TS (10-12). The results of these studies demonstrated that TS overexpression is closely related to the occurrence of 5-FU resistance (13). Thus, TS is not only considered to be a target of 5-FU but also an oncogene participating in 5-FU resistance (14).

Recent studies indicated that prolonged exposure to 5-FU could activate several signaling pathways, including the PI3K/Akt pathway, which is a major downstream effector pathway leading to chemoresistance $(15,16)$. This pathway is involved in cell growth and drug resistance. Recent evidence indicates that activation of the PI3K/AKT pathway contributes to resistance to multiple cancer therapies and is deemed a poor prognostic factor for cancers (17).

Combination studies are widely used in treating dreadful diseases, including cancer (18), and aim to achieve synergistic therapeutic effects, minimize toxicity, and delay the induction of drug resistance. Several compounds from nature, such as medicinal plants, are pharmacologically safe and have been demonstrated to be potent chemosensitizers in combination with conventional chemotherapeutic drugs. Therefore, phytochemicals have a good application prospect in the treatment of cancer and adjuvant chemotherapy (19-22).

Kaempferol is an ideal chemosensitizer owing to its diverse pharmacological actions and nontoxic nature. Fig. 1 illustrates the structure of kaempferol, which is known to exert antitumor effects in various cancer models (23-26). However, no study has been conducted on the effect of 5-FU and kaempferol in cancer. The purpose of this study was to investigate the synergistic antitumor effects of 5-FU and kaempferol in CRC and elucidate the possible mechanisms underlying this effect.

\section{Materials and methods}

Chemicals and reagents. Roswell Park Memorial Institute (RPMI) 1640 medium, fetal bovine serum (FBS), penicillin-streptomycin, trypsin-EDTA, and BCA Protein Assay Kit were obtained from Thermo Fisher Scientific, Inc. An Annexin V-FITC Apoptosis Detection Kit was purchased from Nanjing KeyGen Biotech Co., Ltd. TRIzol Reagent and PrimeScript RT Reagent Kit were provided by Takara Bio, Inc. 5-FU, kaempferol, 3-(4,5-dimethylthiazol-2-yl)-2,5-diphenyl tetrazolium bromide (MTT), and the remaining chemicals used in the present study, unless otherwise stated, were obtained from Sigma-Aldrich; Merck KGaA. Primary antibodies for Bax, Bcl-2, TS, PI3K, Akt, and phosphorylated (p)-Akt and $\beta$-actin horseradish peroxidase (HRP)-conjugated secondary antibodies were provided by Cell Signaling Technology, Inc.

Cell line and cell culture. The CRC cell lines HCT-8, HCT-116 and the normal human embryonic kidney cell line 293 were purchased from the Type Culture Collection of the Chinese Academy of Sciences. The cells were maintained in RPMI-1640 medium supplemented with $10 \%$ FBS, 100 unit $/ \mathrm{ml}$ benzyl penicillin, and $100 \mu \mathrm{g} / \mathrm{ml}$ streptomycin in $5 \% \mathrm{CO}_{2}$ and $95 \%$ air at $37^{\circ} \mathrm{C}$.

Cell viability assay. The HCT- 8 cells and 293 cells $\left(6 \times 10^{3}\right.$ cells/well) were seeded in 96-well plates and exposed to serial dilutions of 5-FU and/or kaempferol for $24 \mathrm{~h}$. Then, MTT reagent $(0.5 \mathrm{mg} / \mathrm{ml}$ in PBS) was added, and the cells were cultured for a further $4 \mathrm{~h}$ at $37^{\circ} \mathrm{C}$ prior to the addition of dimethyl sulfoxide. Absorbance at $570 \mathrm{~nm}$ was measured using an ELISA reader (Model ELX800; BioTek Instruments, Inc.). All assays were independently performed in triplicates. The cell inhibition ratio was calculated as follows: $\left(\mathrm{A}_{\text {control }}-\mathrm{A}_{\text {treated }}\right) / \mathrm{A}_{\text {control }} \mathrm{x} 100 \%$, where $\mathrm{A}_{\text {treated }}$ and $\mathrm{A}_{\text {control }}$ were the absorbance from treated and control groups, respectively. The $\mathrm{IC}_{50}$ values (dose of 5-FU and kaempferol required to inhibit cell growth by $50 \%$ ) were assessed using nonlinear regression analysis.

Calculation of combination index (CI). During the different dose combinations (ratios of $\mathrm{IC}_{50}$ as 5-FU: kaempferol: 2:1, 1:1, 1:2, and 1:4), the HCT-8 and 293 cells were treated with various concentrations of kaempferol and 5-FU; a new concentration-dependent curve was constructed using the MTT assay. In different combination ratios, we used the chessboard concentration dilution method to design drug combinations of different concentrations and then calculated the CI according to the dose-effect curve. The Chou-Talalay method for drug combination is based on the median-effect equation (27,28). Based on these algorithms, CalcuSyn was used for determining synergism and antagonism at all doses or effect levels. The CI was analyzed by CalcuSyn where values $<1,=1$, and $>1$ indicated synergism, an additive effect, and antagonism, respectively.

Identification of apoptosis by Annexin V/propidium iodide (PI) staining. The percentages of cells undergoing apoptosis with or without 5-FU and/or kaempferol were assessed by Annexin V-FITC/PI kit-based FACS (BD Biosciences). Cells were plated $\left(3 \times 10^{5}\right.$ cells/well) in a 6 -well plate and then incubated for $48 \mathrm{~h}$ with $100 \mu \mathrm{M}$ kaempferol or $50 \mu \mathrm{M} 5-\mathrm{FU}$ alone or in combination. Subsequently, the cells were washed twice with cold PBS and stained with Annexin V/PI before being analyzed by FACS, according to the manufacturer's instructions. All assays were performed independently in triplicates.

Hoechst staining. HCT-8 cells were grown in a 6-well plate and treated with $100 \mu \mathrm{M}$ kaempferol and/or $50 \mu \mathrm{M}$ 5-FU for $48 \mathrm{~h}$. The cells were fixed in ice-cold $4 \%$ paraformaldehyde for $10 \mathrm{~min}$. Following washing with PBS, the cells were incubated with $1 \mu \mathrm{g} / \mathrm{ml}$ of Hoechst 33258 solution for $5 \mathrm{~min}$ in the dark. The cells were washed with PBS again and observed under a fluorescent microscope (DMI4000B; Leica Microsystems); the apoptotic cells appeared condensed and displayed fragmented nuclei.

Western blot analysis. Total protein extracts were obtained using lysis buffer and concentrations were determined by the BCA assay (both from Pierce Chemical Co.; Thermo Fisher Scientific, Inc.). Equal amounts of protein $(50 \mu \mathrm{g})$ from each sample were separated by $12 \%$ SDS-PAGE gels and transferred 


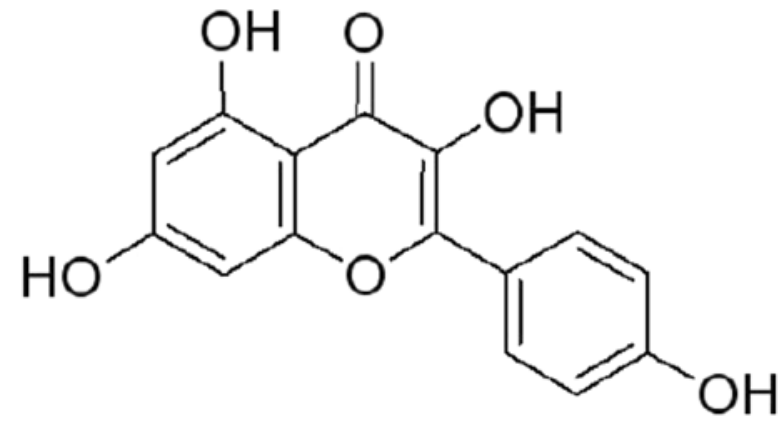

Figure 1. The structure of kaempferol.

to PVDF membranes (EMD Millipore). The membranes were blocked with $5 \%$ skimmed milk for $1 \mathrm{~h}$ at room temperature and probed with specific a primary antibody $\operatorname{Bax}(1: 1,000$; cat. no. 5023, Cell Signaling Technology, Inc.), Bcl-2 (1:1,000; cat. no. 4223; Cell Signaling Technology, Inc.), TS (1:1,000; cat. no. 5449; Cell Signaling Technology, Inc.), $\beta$-actin (1:1,000; cat. no. 4967, Cell Signaling Technology, Inc.), PI3K (1:1,000; cat. no. 4257; 1:1,000), AKT (1:1,000; cat. no. 2938; Cell Signaling Technology, Inc.), p-Akt (1:1,000; ser473; cat. no. 4060, Cell Signaling Technology, Inc.), PTEN (1:1,000; cat. no. 4257; Cell Signaling Technology, Inc.) overnight at $4^{\circ} \mathrm{C}$. After being washed three times with TBST, the membranes were incubated with horseradish peroxidase-conjugated goat anti-rabbit IgG secondary antibody (1:25,000; cat. no. 31460; Thermo Fisher Scientific, Inc.) or rabbit anti-mouse immunoglobulin G secondary antibody (1:25,000; cat. no. 27025; Thermo Fisher Scientific Inc.) at room temperature for $2 \mathrm{~h}$. Following washing again in TBST, protein signals were visualized by an enhanced chemiluminescence reaction system (Bio-Rad Laboratories, Inc.) and quantified using the ImageQuant software (Version 3.0; Bio-Rad Laboratories, Inc.). $\beta$-actin served as the loading control. All protein quantifications were normalized to their respective $\beta$-actin expression levels.

Statistical analysis. Three independent experiments were performed in triplicate. Data are presented as the mean \pm standard deviation (SD). Differences between two groups were analyzed using unpaired Student's t-test. Datasets that involved more than two groups were assessed with one-way analysis variance (ANOVA), along with the Tukey-Kramer test. A P-value $<0.05$ was regarded as statistically significant. Regular analysis was carried out using the SPSS package for Windows (version 17.0; SPSS, Inc.).

\section{Results}

5-FU and kaempferol cause greater inhibition of cell viability in CRC cells. Firstly, the cell viability inhibition potential of each drug was examined in the HCT-8 and HCT-116 cells. As anticipated, the growth of the cells was significantly decreased by treatment with kaempferol and 5-FU in a dose-dependent manner. The $\mathrm{IC}_{50}$ values of 5-FU and kaempferol were 177.78 and $350 \mu \mathrm{M}$, respectively, in HCT-8 cells and 77.63 and $184.33 \mu \mathrm{M}$, respectively, in the HCT-116 cells. The $\mathrm{IC}_{50}$ concentrations were then used to generate fixed ratios for subsequent
Table I. CI for different ratios of 5-FU and kaempferol on HCT-8 cells.

\begin{tabular}{lcc}
\hline Ratio (5-FU: kaempferol) & CI & Effect \\
\hline $1: 2$ & 0.351 & Synergistic \\
$1: 1$ & 0.378 & Synergistic \\
$2: 1$ & 0.808 & Synergistic \\
$4: 1$ & 0.800 & Synergistic \\
\hline
\end{tabular}

CI, combination index; 5-FU, 5-fluorouracil.

Table II. CI for different ratios of 5-FU and kaempferol on HCT-116 cells.

\begin{tabular}{lcc}
\hline Ratio (5-FU: kaempferol) & CI & Effect \\
\hline $1: 10$ & 0.828 & Synergistic \\
$1: 5$ & 0.621 & Synergistic \\
$1: 2.5$ & 0.716 & Synergistic \\
$1: 1.25$ & 0.895 & Synergistic \\
\hline
\end{tabular}

CI, combination index; 5-FU, 5-fluorouracil.

Table III. CI for different ratios of 5-FU and kaempferol on 293 cells

\begin{tabular}{lcc}
\hline Ratio (5-FU: kaempferol) & CI & Effect \\
\hline $12.5: 25$ & 2.852 & Antagonistic \\
$25: 50$ & 1.574 & Antagonistic \\
$50: 100$ & 4.039 & Antagonistic \\
$100: 200$ & 0.895 & Antagonistic \\
\hline
\end{tabular}

CI, combination index; 5-FU, 5-fluorouracil.

combination studies and to calculate the CI. Among them, $50 \mu \mathrm{M}$ of 5 -FU combined with $100 \mu \mathrm{M}$ of kaempferol exhibited synergistic anticancer effects on HCT-8 cells (CI value, 0.351; Table I), when compared with the effects of the two compounds used alone. Consistent results were found in the HCT-116 cell line (CI, 0.621; Table II).

The combination of 5-FU and kaempferol has no synergistic effect on 293 cells. The combination of $100 \mu \mathrm{M}$ of kaempferol with $50 \mu \mathrm{M}$ of 5-FU did not exhibit greater cytotoxic effects on the 293 cells $(\mathrm{CI},>1)$ when compared with either of the two agents used alone (Table III).

The combination of 5-FU and kaempferol exhibits greater inhibition on cell growth and cell viability. Microscopy was used to observe the cell morphology. Cells treated with 5-FU and kaempferol were crenulated, and the nuclei were $\operatorname{dim}$ (Fig. 2A). Cell viability was significantly lower in cells subjected to treatment with 5-FU and kaempferol alone when 
A Control

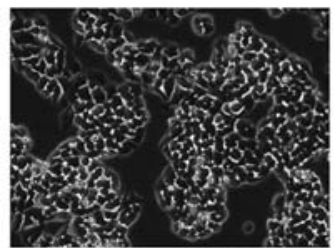

Kaempferol $(100 \mu \mathrm{M})$

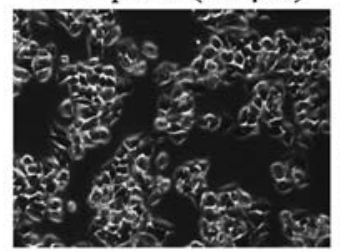

$5-\mathrm{FU}(50 \mu \mathrm{M})$

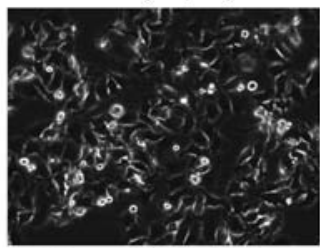

Kaempferol+5-FU

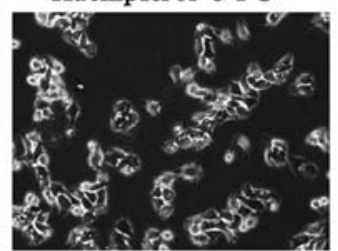

B

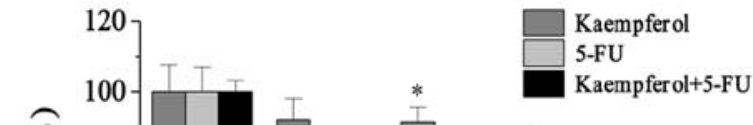

Kaempferol+5-FU

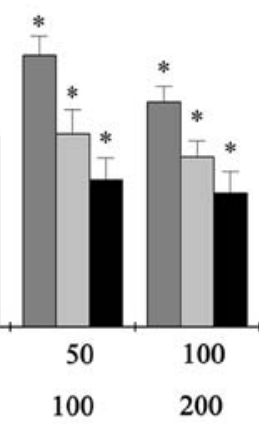

Figure 2. Combination of 5-FU and kaempferol has synergistic effects on the growth and viability of HCT-8 cells. (A) Effect of 5-FU and kaempferol on HCT-8 cell morphology. HCT-8 cells were treated with 5-FU and kaempferol for $48 \mathrm{~h}$, and morphological changes were observed using phase-contrast microscopy. (B) The HCT-8 cells were treated with kaempferol and 5-FU. Relative cell viability was determined as the percentage of absorbance relative to the untreated controls. Data are presented as the mean \pm SD of three independent experiments. ${ }^{*} \mathrm{P}<0.05$ vs. untreated control cells. 5-FU, 5-fluorouracil; SD, standard deviation.

compared with that of the untreated control cells (Fig. 2B). These results indicated that combined treatment with kaempferol and 5-FU could inhibit the growth of HCT-8 cells.

Enhancement of 5-FU-induced apoptosis by kaempferol. To assess if kaempferol plus 5-FU could induce apoptosis, Hoechst staining and flow cytometric analysis were performed to detect cell apoptosis (Fig. 3A). Kaempferol combined with 5-FU treatment significantly induced cancer cell apoptosis in HCT-8 cells when compared with either of the two compounds alone. Although $50 \mu \mathrm{M}$ of 5 -FU and $100 \mu \mathrm{M}$ of kaempferol induced 10.13 and $3.31 \%$ apoptosis, respectively, in HCT- 8 cells, a combination of these two induced $31.41 \%$ apoptosis, which is almost three times that induced by $50 \mu \mathrm{M}$ 5-FU (Fig. 3B).

Kaempferol combined with 5-FU upregulates the expression levels of apoptosis-associated proteins Bax, Bcl-2, and 5-FU metabolic enzyme TS. Apoptosis-related gene expression of Bax, Bcl-2, and 5-FU metabolic enzyme TS was detected by western blotting. The expression levels of Bax were higher in cells subjected to combination treatment when compared with those with either agent alone (Fig. 4). Conversely, the expression levels of Bcl-2 were decreased in the combination group when compared with single-agent treatment; TS expression levels were significantly decreased in HCT-8 cells when treated with kaempferol and 5-FU (Fig. 4). These results indicated that kaempferol combined with 5-FU exhibits synergistic anticancer effects by inducing CRC cell apoptosis and altering the expression levels of TS.

Role of the PI3K/AKT pathway in the synergistic effects of kaempferol and 5-FU. To determine whether AKT activation was involved in the synergistic effects of kaempferol and 5-FU, the levels of PI3K, PTEN, AKT, and p-AKT in HCT- 8 cells were examined by western blotting. The p-AKT levels were attenuated in cells treated with kaempferol and increased after 5-FU treatment. However, PI3K and p-AKT levels were significantly lower in cells subjected to combination treatment when compared with 5-FU alone (Fig. 5). Thus, kaempferol and 5-FU may have synergistically suppressed CRC cell growth by inhibiting the activation of the PI3K/Akt pathway.

\section{Discussion}

Chemotherapy is considered as the most potent treatment option to improve poor survival rates in cancer. Although the combination of 5-FU, oxaliplatin, and irinotecan are being used in the clinical setting, their effects are not entirely satisfactory (2). The two main problems associated with chemotherapy are drug toxicity and the development of resistance of the tumor cells toward apoptosis. Thus, the combination of 5-FU with chemosensitizers could minimize the occurrence of side effects and maximize efficacy. Several synthetic chemosensitizers have been developed, but their cytotoxic effects and adverse pharmacokinetics have prohibited their use in clinical trials.

Hedyotis diffusa Willd is a major component frequently used in traditional Chinese medicine for the clinical treatment of CRC and is associated with drug resistance $(29,30)$. Kaempferol is one of the main active components of Hedyotis diffusa and has been revealed to possess anticancer effects in several cancer cell lines both in vitro and in vivo (31-36). Notably, they exhibit almost no or minor toxicity against normal epithelial, peripheral blood, and myeloid cells. In the present study, the effects of different combinations of kaempferol and 5-FU were examined; the inhibition rates were analyzed by the method described by Chou and Talalay. As revealed in Table I, the combined inhibitory effect of kaempferol and 5-FU $(\mathrm{CI},<1)$ on the growth of the CRC cells was stronger than that of kaempferol or 5-FU alone. The following combination was used for further evaluations and comparisons in this study: kaempferol $(100 \mu \mathrm{M})$ and 5-FU (50 $\mu \mathrm{M})$.

The effect of kaempferol $(100 \mu \mathrm{M})$ and 5-FU (50 $\mu \mathrm{M})$ on apoptosis induction in HCT-8 cells was higher than that of either of the agents used alone. These results encouraged further evaluations into the mechanism of this synergistic effect. 
A

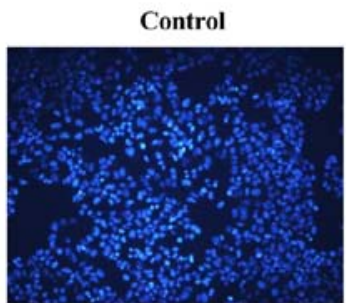

B

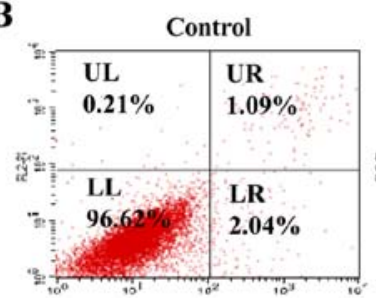

\section{Kaempferol (100 $\mu \mathrm{M})$}

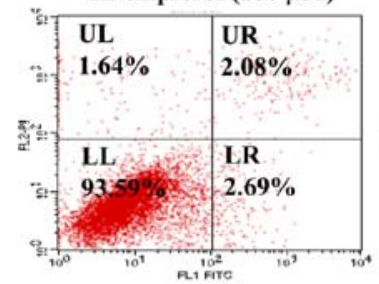

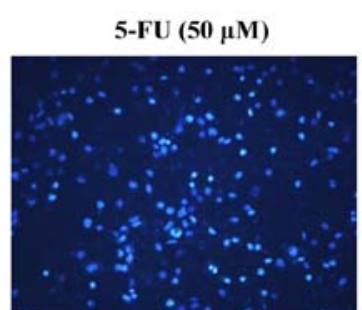

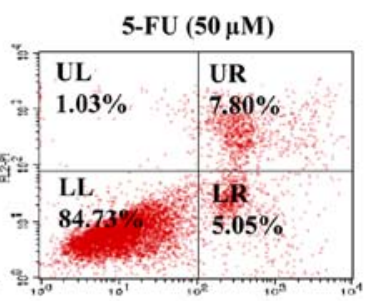

Kaempferol+5-FU

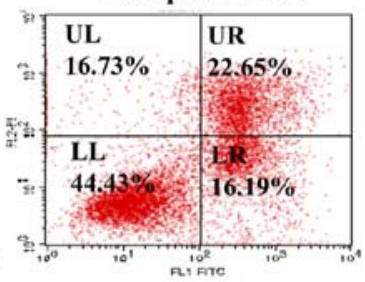

Kaempferol (100 $\mu \mathrm{M})$
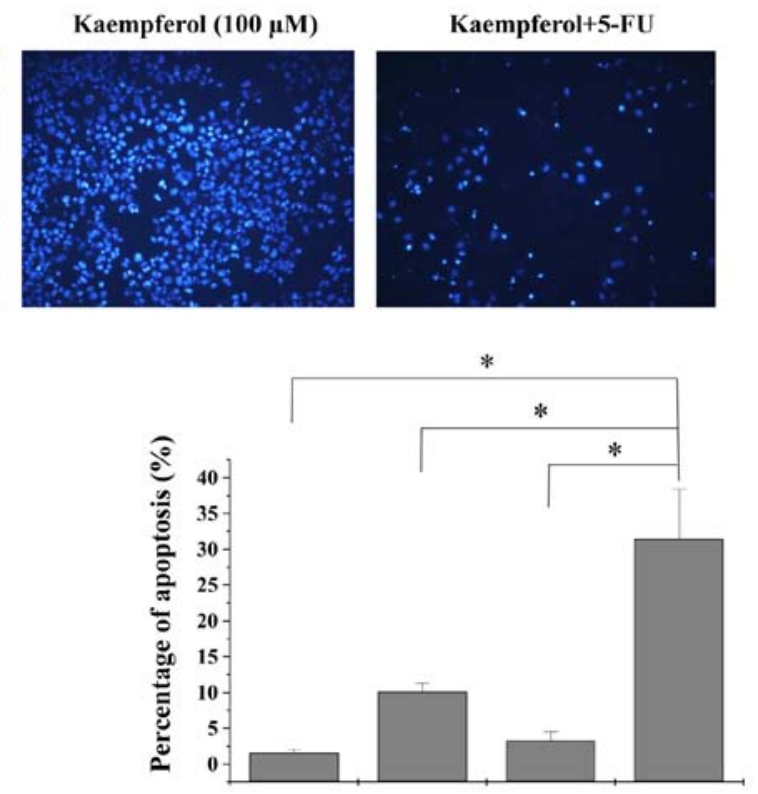

5-FU $(\mu M) \quad-\quad 50$

-
50

100

Figure 3. The enhancement of 5-FU-induced apoptosis by kaempferol. (A) HCT-8 cells were treated with 5-FU, kaempferol, or a combination of both for $48 \mathrm{~h}$. The cells were cultured in fresh medium for Hoechst staining assay. (B) Flow cytometry was used to detect apoptosis in HCT-8 cells treated with 5-FU and/or kaempferol for $48 \mathrm{~h}$. Annexin V/PI double-negative population indicates viable cells, and Annexin V-positive/PI-negative or Annexin V/PI double-positive population represents cells undergoing early or late apoptosis, respectively. Data are representative of at least three independent experiments, which are presented as the mean \pm SD of triplicate experiments. "P<0.05 vs. untreated control cells. 5 -FU, 5 -fluorouracil; SD, standard deviation.
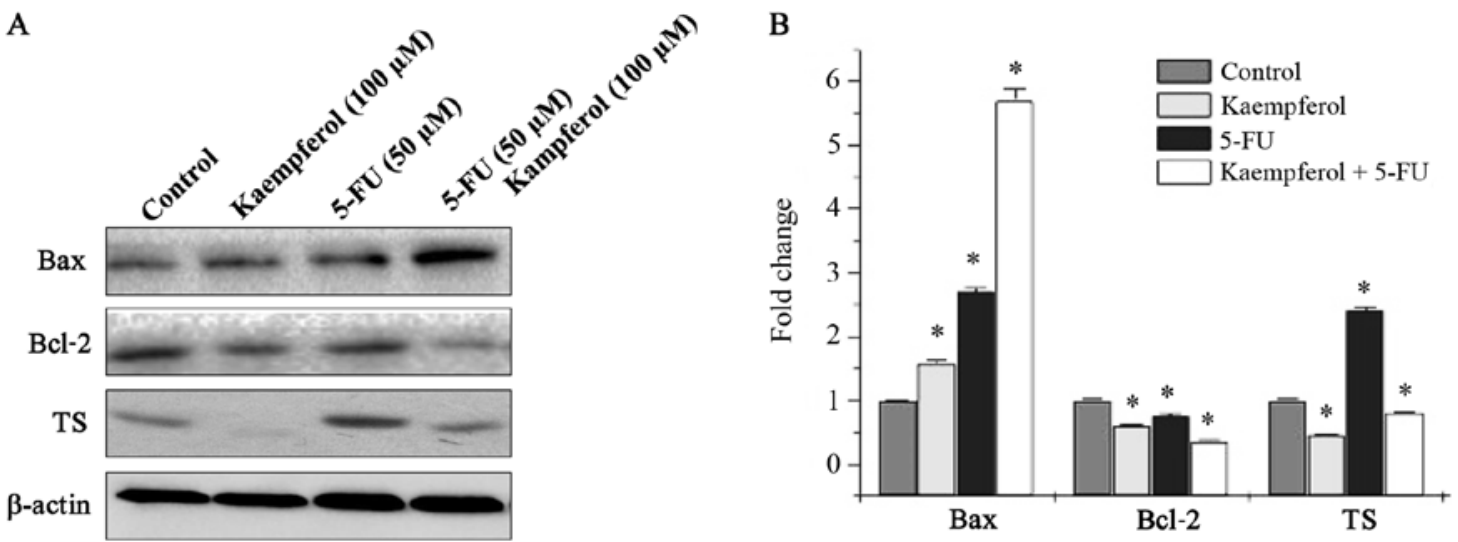

Figure 4. Kaempferol combined with 5-FU regulates the protein expression levels of Bax, Bcl-2, and TS. (A) Bax, Bcl-2, and TS protein expression levels in HCT-8 cells were assessed by western blot analysis. (B) Quantitation of the expression levels by densitometric analysis. $\beta$-actin served as the loading control. All protein quantifications were normalized to their respective $\beta$-actin expression levels. Data are representative of at least three independent experiments. ${ }^{*} \mathrm{P}<0.05$ vs. untreated control cells. 5-FU, 5-fluorouracil; TS, thymidylate synthase.

It is widely accepted that the PI3K/Akt pathway plays an important role in drug resistance. Overexpression of this PI3K/Akt pathway has been identified in 5-FU-resistant cell lines, and the blocking of this pathway could sensitize cancer cells to 5-FU in vitro (37). However, activation of the PI3K/Akt pathway was revealed to induce 5-FU resistance in cancer cells (38). Furthermore, this pathway has a major function in cell proliferation and apoptosis. Apoptosis-related proteins, such as Bax, Bcl-2, and the 5-FU metabolic enzyme TS, are major downstream effectors of the PI3K/Akt signaling pathway (39).
The intrinsic apoptotic pathway is largely controlled by proapoptotic (Bax) and the antiapoptotic (Bcl-2) proteins (40). In the present study, kaempferol combined with 5-FU decreased the expression levels of Bcl-2 and Bax when compared with kaempferol or 5-FU alone. Thus, based on the results of Hoechst nuclear staining, and flow cytometric and western blot analyses, the synergistic effect of 5-FU and kaempferol on apoptosis induction was confirmed. The formation of the apoptosome causes cleavage of procaspases (caspase family) which are responsible for activating effector caspases, such as caspase-3, which is a key protease of the apoptotic machinery and ultimately resulting in 


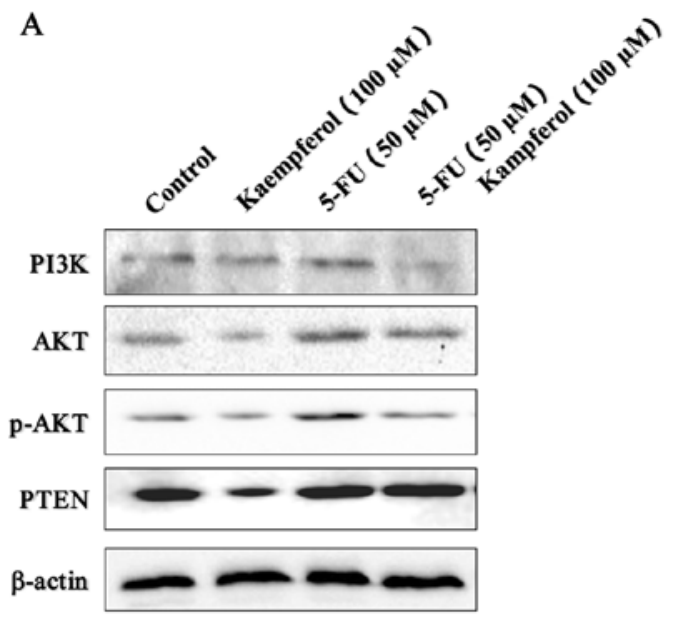

B

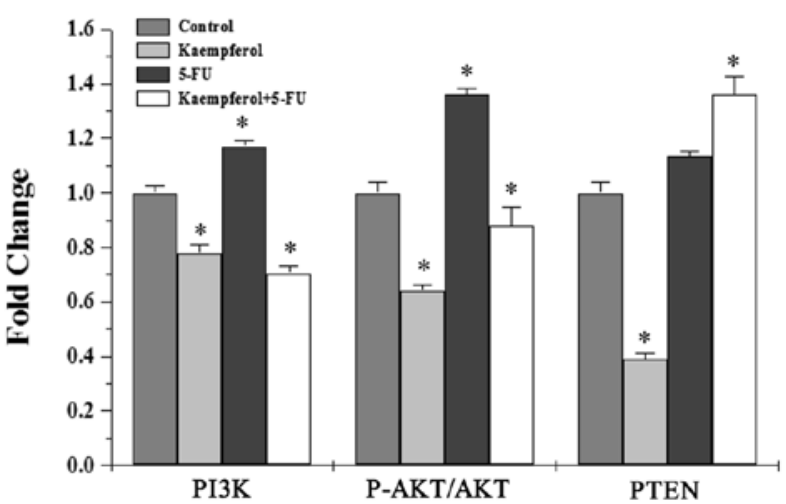

Figure 5. Role of the PI3K/Akt pathway in the synergistic effects of kaempferol and 5-FU. (A) PI3K, AKT, p-AKT, and PTEN protein expression levels in HCT-8 cells were assessed by western blot analysis. (B) Quantitation of the protein expression levels by densitometric analysis. $\beta$-actin served as the loading control. All protein quantifications were normalized to their respective $\beta$-actin expression levels. Data are representative of at least three independent experiments. "P<0.05 vs. untreated control cells. 5-FU, 5-fluorouracil; p-AKT, phosphorylated AKT.

apoptosis (41). Among them, it is unclear how the synergy of kaempferol and 5-FU affects the caspase family and ultimately promotes apoptosis. In addition, it was also revealed that combination of 5-FU and kaempferol could arrest the cell cycle in the $\mathrm{S}$ phase, while the specific regulatory mechanism of kaempfetol is unknown. All of these issues rquire further study.

TS, a critical 5-FU-targeted enzyme, participates in 5-FU resistance in cancer patients receiving chemotherapy. TS has been well accepted as one of the most important targets of 5-FU resistance (42). A previous study indicated that TS was dramatically increased following prolonged exposure to 5-FU (43). Consistent with previous research, the expression level of TS was increased along with the decrease in 5-FU sensitivity after 5-FU treatment in the present study. Additionally, TS levels could be downregulated by kaempferol; thus, kaempferol may increase 5-FU sensitivity by upregulating the expression levels of TS, thereby contributing to the synergistic effects of kaempferol and 5-FU.

In the present study, kaempferol combined with 5-FU demonstrated synergistic anticancer effects by inducing apoptosis and altering the expression levels of TS in CRC cells. These effects may have occurred via attenuation of the activation of p-AKT and suppression of the PI3K/AKT pathway.

\section{Acknowledgements}

Not applicable.

\section{Funding}

No funding was received.

\section{Availability of data and materials}

The datasets used and/or analyzed during the current study are available from the corresponding author on reasonable request.

\section{Authors' contributions}

JP designed the present study. QL, LW, SL performed the experiments. QL, JL and YC analyzed the data. QL and JL and $\mathrm{YC}$ wrote the paper. All authors read and approved the final manuscript.

\section{Ethics approval and consent to participate}

Not applicable.

\section{Patient consent for publication}

Not applicable.

\section{Competing interests}

The authors declare that they have no competing interests.

\section{References}

1. Miller KD, Siegel RL, Lin CC, Mariotto AB, Kramer JL, Rowland JH, Stein KD, Alteri R and Jemal A: Cancer treatment and survivorship statistics, 2016. CA Cancer J Clin 66: 271-289, 2016.

2. O'Dwyer PJ, Eckhardt SG, Haller DG, Tepper J, Ahnen D, Hamilton S, Benson AB III, Rothenberg M, Petrelli N, Lenz HJ, et al: Priorities in colorectal cancer research: Recommendations from the gastrointestinal scientific leadership council of the coalition of cancer cooperative groups. J Clin Oncol 25: 2313-2321, 2007.

3. Mody K and Bekaii-Saab T: Clinical trials and progress in metastatic colon cancer. Surg Oncol Clin N Am 27: 349-365, 2018.

4. Montagnani F, Chiriatti A, Turrisi G, Francini G and Fiorentini G: A systematic review of FOLFOXIRI chemotherapy for the first-line treatment of metastatic colorectal cancer: Improved efficacy at the cost of increased toxicity. Colorectal Dis 13: 846-852, 2011.

5. Kanazawa Y, Yamada T, Fujita I, Kakinuma D, Matsuno K, Arai H, Shimoda T, Ko K, Kato S, Matsutani T, et al: In vitro chemosensitivity test for gastric cancer specimens predicts effectiveness of oxaliplatin and 5-fluorouracil. Anticancer Res 37: 6401-6405, 2017. 
6. Pardini B, Kumar R, Naccarati A, Novotny J, Prasad RB, Forsti A, Hemminki K, Vodicka P and Lorenzo Bermejo J: 5-Fluorouracil-based chemotherapy for colorectal cancer and MTHFR/MTRR genotypes. Br J Clin Pharmacol 72: 162-163, 2011.

7. Ghoshal K and Jacob ST: An alternative molecular mechanism of action of 5-fluorouracil, a potent anticancer drug. Biochem Pharmacol 53: 1569-1575, 1997.

8. Latchman J, Guastella A and Tofthagen C: 5-fluorouracil toxicity and dihydropyrimidine dehydrogenase enzyme: Implications for practice. Clin J Oncol Nurs 18: 581-585, 2014.

9. Papanastasopoulos P and Stebbing J: Molecular basis of 5-fluorouracil-related toxicity: Lessons from clinical practice. Anticancer Res 34: 1531-1535, 2014.

10. Wang W, McLeod HL, Cassidy J and Collie-Duguid ES: Mechanisms of acquired chemoresistance to 5-fluorouracil and tomudex: Thymidylate synthase dependent and independent networks. Cancer Chemother Pharmacol 59: 839-845, 2007.

11. Sigmond J, Backus HH, Wouters D, Temmink OH, Jansen G and Peters GJ: Induction of resistance to the multitargeted antifolate Pemetrexed (ALIMTA) in WiDr human colon cancer cells is associated with thymidylate synthase overexpression. Biochem Pharmacol 66: 431-438, 2003.

12. Peters GJ, Backus HH, Freemantle S, van Triest B, Codacci-Pisanelli G, van der Wilt CL, Smid K, Lunec J, Calvert AH, Marsh S, et al: Induction of thymidylate synthase as a 5-fluorouracil resistance mechanism. Biochim Biophys Acta 1587: 194-205, 2002.

13. Etienne MC, Chazal M, Laurent-Puig P, Magné $N$, Rosty C, Formento JL, Francoual M, Formento P, Renée N, Chamorey $\mathrm{E}$, et al: Prognostic value of tumoral thymidylate synthase and p53 in metastatic colorectal cancer patients receiving fluorouracil-based chemotherapy: Phenotypic and genotypic analyses. J Clin Oncol 20: 2832-2843, 2002.

14. Rahman L, Voeller D, Rahman M, Lipkowitz S, Allegra C, Barrett JC, Kaye FJ and Zajac-Kaye M: Thymidylate synthase as an oncogene: A novel role for an essential DNA synthesis enzyme. Cancer Cell 5: 341-351, 2004

15. Cassinelli G, Zuco V, Gatti L, Lanzi C, Zaffaroni N, Colombo D and Perego P: Targeting the Akt kinase to modulate survival, invasiveness and drug resistance of cancer cells. Curr Med Chem 20: 1923-1945, 2013

16. Das D, Satapathy SR, Siddharth S, Nayak A and Kundu CN: NECTIN-4 increased the 5-FU resistance in colon cancer cells by inducing the PI3K-AKT cascade. Cancer Chemother Pharmacol 76: 471-479, 2015

17. Xu J, Zhang S, Wang R, Wu X, Zeng L and Fu Z: Knockdown of PRDX2 sensitizes colon cancer cells to 5-FU by suppressing the PI3K/AKT signaling pathway. Biosci Rep 37: pii: BSR20160447, 2017.

18. Das R, Bhattacharya K, Samanta SK, Pal BC and Mandal C: Improved chemosensitivity in cervical cancer to cisplatin: Synergistic activity of mechanism through STAT3 inhibition. Cancer Lett 351: 81-90, 2014

19. Li CJ, Chu CY, Huang LH, Wang MH, Sheu LF, Yeh JI and Hsu HY: Synergistic anticancer activity of triptolide combined with cisplatin enhances apoptosis in gastric cancer in vitro and in vivo. Cancer Lett 319: 203-213, 2012.

20. Vinod BS, Antony J, Nair HH, Puliyappadamba VT, Saikia M, Narayanan SS, Bevin A and Anto RJ: Mechanistic evaluation of the signaling events regulating curcumin-mediated chemosensitization of breast cancer cells to 5-fluorouracil. Cell Death Dis 4 e505, 2013

21. Lee YJ, Lee S, Ho JN, Byun SS, Hong SK, Lee SE and Lee E: Synergistic antitumor effect of ginsenoside Rg3 and cisplatin in cisplatin-resistant bladder tumor cell line. Oncol Rep 32: 1803-1808, 2014

22. Bi T, Zhu A, Yang X, Qiao H, Tang J, Liu Y and Lv R: Metformin synergistically enhances antitumor activity of cisplatin in gallbladder cancer via the PI3K/AKT/ERK pathway. Cytotechnology 70: 439-448, 2018.

23. Li C, Zhao Y, Yang D, Yu Y, Guo H, Zhao Z, Zhang B and Yin X: Inhibitory effects of kaempferol on the invasion of human breast carcinoma cells by downregulating the expression and activity of matrix metalloproteinase-9. Biochem Cell Biol 93: 16-27, 2015.

24. Hung TW, Chen PN, Wu HC, Wu SW, Tsai PY, Hsieh YS and Chang HR: Kaempferol inhibits the invasion and migration of renal cancer cells through the downregulation of AKT and FAK pathways. Int J Med Sci 14: 984-993, 2017.
25. Huang WW, Tsai SC, Peng SF, Lin MW, Chiang JH, Chiu YJ, Fushiya S, Tseng MT and Yang JS: Kaempferol induces autophagy through AMPK and AKT signaling molecules and causes G2/M arrest via downregulation of CDK1/cyclin B in SK-HEP-1 human hepatic cancer cells. Int J Oncol 42: 2069-2077, 2013.

26. Feng J, Jin Y, Peng J, Wei L, Cai Q, Yan Z, Lai Z and Lin J: Hedyotis diffusa willd extract suppresses colorectal cancer growth through multiple cellular pathways. Oncol Lett 14: 8197-8205, 2017

27. Chou TC: Synergy determination issues. J Virol 76: 10577-10578, 2002.

28. Chou TC: Drug combination studies and their synergy quantification using the Chou-Talalay method. Cancer Res 70: 440-446, 2010.

29. Li Q, Lai Z, Yan Z, Peng J, Jin Y, Wei L and Lin J: Hedyotis diffusa Willd inhibits proliferation and induces apoptosis of 5-FU resistant colorectal cancer cells by regulating the PI3K/AKT signaling pathway. Mol Med Rep 17: 358-365, 2018.

30. Li Q, Wang X, Shen A, Zhang Y, Chen Y, Sferra TJ, Lin J and Peng J: Hedyotis diffusa Willd overcomes 5-fluorouracil resistance in human colorectal cancer HCT-8/5-FU cells by downregulating the expression of $\mathrm{P}$-glycoprotein and ATP-binding casette subfamily G member 2. Exp Ther Med 10: 1845-1850, 2015

31. Diantini A, Subarnas A, Lestari K, Halimah E, Susilawati Y, Supriyatna, Julaeha E, Achmad TH, Suradji EW, Yamazaki C, et al: Kaempferol-3-O-rhamnoside isolated from the leaves of Schima wallichii Korth. inhibits MCF-7 breast cancer cell proliferation through activation of the caspase cascade pathway. Oncol Lett 3 : 1069-1072, 2012

32. Luo H, Rankin GO, Li Z, Depriest L and Chen YC: Kaempferol induces apoptosis in ovarian cancer cells through activating p53 in the intrinsic pathway. Food Chem 128: 512-519, 2011.

33. Jo E, Park SJ, Choi YS, Jeon WK and Kim BC: Kaempferol suppresses transforming growth factor- $\beta 1$-induced epithelial-to-mesenchymal transition and migration of A549 lung cancer cells by inhibiting Akt1-mediated phosphorylation of Smad3 at threonine-179. Neoplasia 17: 525-537, 2015.

34. Tu LY, Bai HH, Cai JY and Deng SP: The mechanism of kaempferol induced apoptosis and inhibited proliferation in human cervical cancer SiHa cell: From macro to nano: From macro to nano. Scanning 38: 644-653, 2016

35. Wu LY, Lu HF, Chou YC, Shih YL, Bau DT, Chen JC, Hsu SC and Chung JG: Kaempferol induces DNA damage and inhibits DNA repair associated protein expressions in human promyelocytic leukemia HL-60 cells. Am J Chin Med 43: 365-382, 2015.

36. Song H, Bao J, Wei Y, Chen Y, Mao X, Li J, Yang Z and Xue Y: Kaempferol inhibits gastric cancer tumor growth: An in vitro and in vivo study. Oncol Rep 33: 868-874, 2015.

37. Ishida K, Ito C, Ohmori Y, Kume K, Sato KA, Koizumi Y, Konta A, Iwaya T, Nukatsuka M, Kobunai T, et al: Inhibition of PI3K suppresses propagation of drug-tolerant cancer cell subpopulations enriched by 5-fluorouracil. Sci Rep 7: 2262, 2017.

38. Kim EJ, Kang GJ,Kang JI, Boo HJ, Hyun JW, Koh YS, Chang WY, Kim YR, Kwon JM, Maeng YH, et al: Over-activation of AKT signaling leading to 5-Fluorouracil resistance in SNU-C5/5-FU cells. Oncotarget 9: 19911-19928, 2018.

39. Nagaraju GP, Alese OB, Landry J, Diaz R and El-Rayes BF HSP90 inhibition downregulates thymidylate synthase and sensitizes colorectal cancer cell lines to the effect of 5FU-based chemotherapy. Oncotarget 5: 9980-9991, 2014.

40. Kang MH and Reynolds CP: Bcl-2 inhibitors: Targeting mitochondrial apoptotic pathways in cancer therapy. Clin Cancer Res 15: 1126-1132, 2009.

41. Larsen BD and Sørensen CS: The caspase-activated DNase: Apoptosis and beyond. FEBS J 284: 1160-1170, 2017.

42. Jiang B, Liu F, Liu Z, Zhang T and Hua D: B7-H3 increases thymidylate synthase expression via the PI3k-Akt pathway. Tumor Biol 37: 9465-9472, 2016.

43. Milczarek M, Rossowska J, Klopotowska D, Stachowicz M, Kutner A and Wietrzyk J: Tacalcitol increases the sensitivity of colorectal cancer cells to 5 -fluorouracil by downregulating the thymidylate synthase. J Steroid Biochem Mol Biol 190: 139-151, 2019. 